\title{
Problems with the sources of the observed gravitational waves and their res- olution
}

\author{
A.D. Dolgov ${ }^{1,2, a}$ \\ ${ }^{1}$ NSU, Novosibirsk, 630090, Russia \\ ${ }^{2}$ ITEP, Moscow, 117218, Russia
}

\begin{abstract}
Recent direct registration of gravitational waves by LIGO and astronomical observations of the universe at redshifts 5-10 demonstrate that the standard astrophysics and cosmology are in tension with the data. The origin of the source of the GW150914 event, which presumably is a binary of coalescing black holes with masses about 30 solar masses, each with zero spin, as well as the densely populated universe at $\mathrm{z}=5-10$ by superheavy black holes, blight galaxies, supernovae, and dust does not fit the standard astrophysical picture. It is shown here that the model of primordial black hole (PBH) formation, suggested in 1993, nicely explains all these and more puzzles, including those in contemporary universe, such as MACHOs and the mass spectrum of the observed solar mass black holes.. The mass spectrum and density of PBH is predicted. The scenario may possibly lead to abundant antimatter in the universe and even in the Galaxy.
\end{abstract}

\section{Introduction}

The standard cosmological $\Lambda$ CDM model (here CMD stands for Cold Dark Matter and $\Lambda$ for vacuum-like dark energy, or, what is the same, $\Lambda$-term) very well describes gross features of the universe such as the spectrum of perturbations at large scales, features of CMB (especially the shape of the angular fluctuation spectrum), baryogenesis, big bang nucleosynthesis, etc at expense of a few parameters. However many ingredients of the standard cosmology are absent in the minimal standard model (MSM) of particle physics, in particular, dark matter, dark energy, baryogenesis, and vacuum energy. To be more precise, the situation with vacuum energy is opposite: there is too much vacuum energy in the MSM. For example the energy of the gluon and quark condensate established by quantum chromodynamics are roughly 45 orders of magnitude higher than the observed magnitude of the dark energy.

So we have to conclude that new physics beyond the frameworks of MSM is a necessity. Still, except for the vacuum energy problem, the new physics may be almost the old one with introduction of some new fields of particles, while it is quite possible that for the solution of the vacuum energy problem a revolutionary modification of the existing theory would be necessary.

In addition to these well known good old problems the last several years revealed many features which look surprising and even completely mysterious in the frameworks of the $\Lambda \mathrm{CDM}$ model. These recent discoveries are reviewed in what follows. It is argued that all them can be explained by formation of heavy primordial black holes (PBHs) through the mechanisms suggested and discussed

\footnotetext{
a e-mail: dolgov@fe.infn.it
}

in detail in papers [1]. This mechanism allows for formation of PBHs with the masses in the range from a fraction of the solar mass, say, up to $10^{4} M_{\odot}$ or even higher. Usually only the PBHs with rather low masses $\sim 10^{20} \mathrm{~g}$ were considered.

Moreover, the puzzling properties of the sources of the gravitational waves recently discovered by LIGO [2] are explained by the same scenario of heavy $\mathrm{PBH}$ formation.

The content of the talk is the following:

1. GW observation by LIGO.

2. Problems with the GW sources.

3. Solution of the problems and predictions.

4. Dense population of the universe at $z \sim 10$ by the objects which could not be there. They include in particularly supermassive BHs, early supernovae and gamma-bursters, evolved chemistry and dust in high $z$ universe.

5. Problems in present day universe: MACHOs, $\mathrm{PBH}$ dark matter, supermassive BHs in large galaxies and even in almost empty space.

The talk is based on several our papers [3-7], where the relevant references can be found.

\section{Direct discovery of gravitations waves}

On February 11, LIGO (Laser Interferometer Gravitational wave Observatory) collaborations announced discovery of gravitational waves from a coalescing binary systems of black holes [2]. Two more events were reported shortly, see below Table. 1 .

The shape of the signal is in perfect agreement with the theory of BH interactions in the strong (Schwarzschild) sefl-fields, so it can be considered as a first direct proof of $\mathrm{BH}$ existence and a persuasive confirmation of General 
Relativity for a large deviation of geometry from the flat Minkowsky one. All the previous tests were about weak fields only.

This discovery opened a new era of gravitational waves telescopes which will presumably allow to observe several (many) such catastrophic events per year. The anticipated increase of the LIGO sensitivity by factor three will give enhance the number of the registered events 27 -fold. With the expected onset of operation of VIRGO (Italy) and KAGRA (The Kamioka Gravitational Wave Detector, Japan) the direction to the sources can be reliably established, so the sources can be studied by optical and other electromagnetic telescopes. New discoveries are imminent.

In Table I, copied from ref. [2], the properties of the two coalescing black holes, which produced the powerful burst of gravitational waves, are presented. The mass and spin of the final $\mathrm{BH}$, and the total energy radiated in gravitational waves are estimated by the fits to numerical simulations of binary black hole mergers. The estimated total energy radiated in gravitational waves is $(3.0 \pm 0.5) M_{\odot}$ and the peak of gravitational-wave luminosity is $3.0_{-0.4}^{+0.5} \times 10^{56} \mathrm{erg} / \mathrm{sec}$ equivalent to $200 M_{\odot} / \mathrm{sec}$, more than whole radiation power of the visible universe. Rotational energy (outside the $\mathrm{BH}$ ) is about $0.3 M_{\odot}$. It may be in principle extracted.

TABLE I. Source parameters for GW150914. We report median values with $90 \%$ credible intervals that include statistical errors, and systematic errors from averaging the results of different waveform models. Masses are given in the source frame; to convert to the detector frame multiply by $(1+z)$ [90]. The source redshift assumes standard cosmology [91].

\begin{tabular}{lc}
\hline \hline Primary black hole mass & $36_{-4}^{+5} M_{\odot}$ \\
Secondary black hole mass & $29_{-4}^{+4} M_{\odot}$ \\
Final black hole mass & $62_{-4}^{+4} M_{\odot}$ \\
Final black hole spin & $0.67_{-0.07}^{+0.05}$ \\
Luminosity distance & $410_{-180}^{+160} \mathrm{Mpc}$ \\
Source redshift $z$ & $0.09_{-0.04}^{+0.03}$ \\
\hline \hline
\end{tabular}

Three GW event observed to the present time are described in Fig. 1. The last event is questionable while the first two look reliable enough. The essential difference between GW150914 and GW151226 is the following: the masses of the sources in the first case are very high, they are are 36 and 29 solar masses, while in the second case they are $14 M_{\odot}$ and $7 M_{\odot}$, which look astrophysically normal. The spins of both progenitors in the first case are low, compatible with zero. In the second case one of the black holes has noticeable spin $a>0.2$. The formation mechanism of the initial back holes in the first case demands some unknown, unusual astrophysical processes, while each of the companions on the second pair may be created through the usual astrophysical channel. However, the formation of the binaries in both cases is not well understood, see below.
There are essentially three problems in the standard theory:

1. Origin of heavy BHs $\left(\sim 30 M_{\odot}\right)$.

2. Low spins of the coalescing BHs.

3. Formation of $\mathrm{BH}$ binaries from original stellar binaries.

The first problem is a heavy $\mathrm{BH}$ origin. Such BHs are believed to be created by massive star collapse, though a convincing theory is still lacking. To form so heavy BHs, the progenitors should have $M>100 M_{\odot}$ and a low metal abundance to avoid too much mass loss during the evolution. Such heavy stars might be present in young starforming galaxies but they are not yet observed in sufficiently high number.

Another problem is the low value of the $\mathrm{BH}$ spins in GW150914. It strongly constrains astrophysical BH formation from close binary systems. However, the dynamical formation of double massive low-spin BHs in dense stellar clusters is not excluded. The second reliable LIGO detection, GW151226, turned out to be closer to the standard binary $\mathrm{BH}$ system.

Last but not the least is the problem of formation of $\mathrm{BH}$ binaries. Stellar binaries were formed from common interstellar gas clouds and are quite frequent in galaxies. If $\mathrm{BH}$ is created through stellar collapse, a small non-sphericity of collapse results in a huge velocity of the $\mathrm{BH}$ and the binary is destroyed. An indirect evidence for that is presented by large velocities of pulsars in the Galaxy. Their velocities are about $1000 \mathrm{~km} / \mathrm{sec}$, while the average star velocities are only $200-300 \mathrm{~km} / \mathrm{sec}$. Moreover, the BH formation from PopIII stars and subsequent formation of $\mathrm{BH}$ binaries with $\sim(30+30) M_{\odot}$ is analyzed in the literature and is found to be negligible.

All these problems are solved if the observed sources of GWs are the binaries of primordial black holes $(\mathrm{PBH})$. Here a model of $\mathrm{PBH}$ formation is presented which naturally reproduces the puzzling properties of GW150914, the rate of binary $\mathrm{BH}$ merging events inferred from the first LIGO science run, and provides seeds for early supermassive $\mathrm{BH}$ formation. These $\mathrm{PBH}$ are created at rest and so their mutual capture to form a binary is not inhibited after they loose their relative velocity due e.g. to dynamical friction. The spins of the original BHs are naturally zero because rotational perturbations are absent in the early universe.

In addition, the mechanism explains an avalanche of mysteries discovered recently and may provide all or a large fraction of cosmological DM in the form of PBHs with rather wide mass spectrum.

\section{Mechanism of massive PBH formation.}

The model of an early BH creation is based on the supersymmetric (Affleck-Dine) scenario for baryogenesis [8], modified by introduction of a general renormalizable coupling to the inflaton field, see below, Eq. (6). It was suggested in 1993 [1] and discussed in more details in several our papers applied to an explanation of existence of the observed "old" objected in the young universe. As a byproduct the model may lead to an abundant antimatter objects in the universe and, in particular, in the Galaxy. 
The basic ingredient of the Affleck-Dine (AD) scenario is a scalar field $\chi$ with non-zero baryonic number, $B \neq 0$. As is well known, scalar baryons must exist in supersymmetric theories. Generically the potential of such field has the so called flat directions along which the potential does not rise. We can take as toy model example the potential of the form:

$$
U_{\lambda}(\chi)=\lambda|\chi|^{4}(1-\cos 4 \theta),
$$

where $\theta$ is the phase of the complex field $\chi=|\chi| \exp (i \theta)$. Such bosons, $\chi$, may condense along flat directions of this potential accumulating large baryonic number. To be more precise, the baryonic number is accumulated not in the large amplitude of $\chi$ but in its angular momentum associated with variation of $\theta(t)$, see eq. (4).

In addition to the quartic potential there may exist quadratic mass term, $m^{2} \chi^{2}+m^{* 2} \chi^{* 2}$, which also could have flat directions generally different from those in quartic poteetial:

$$
U_{m}(\chi)=m^{2}|\chi|^{2}[1-\cos (2 \theta+2 \alpha),],
$$

where $\chi=|\chi| \exp (i \theta)$ and $m=|m| e^{i \alpha}$. If $\alpha \neq 0, \mathrm{C}$ and $\mathrm{CP}$ are broken. In GUT SUSY baryonic number is naturally non-conserved. In out model this non-conservation is induced by non-invariance of $U(\chi)$ w.r.t. the phase rotation of $\chi$.

Initially (after inflation) $\chi$ was naturally away from the origin due to rising quantum fluctuations of light fields. After inflation terminated, $\chi$ started to evolve down to the equilibrium point, $\chi=0$, according to the its equation of motion, which for homogenous field formally coincides with the equation of motion for point-like body in the Newtonian mechanics:

$$
\ddot{\chi}+3 H \dot{\chi}+U^{\prime}(\chi)=0 .
$$

The second term in this equation, induced by the universe expansion, is called the Hubble friction term. In the mechanical analogy it is equivalent to liquid friction.

The baryonic number of $\chi$ is equivalent to the angular momentum of $\chi$-rotation in two dimensional complex plane $[\operatorname{Re} \chi, \operatorname{Im} \chi]$ :

$$
B_{\chi}=\dot{\theta}|\chi|^{2} \text {. }
$$

Later the decays of $\chi$ transferred its baryonic number to baryonic number of quarks in B-conserving process. The Affleck-Dine baryogenesis could lead to the cosmological baryon asymmetry of order of unity, much larger than the observed value $\beta=N_{B} / N_{\gamma} \sim 10^{-9}$.

When inflation terminated and the Hubble friction drastically dropped down, the field $\chi$ started to move to the origin, $\chi=0$, along the flat direction of the quartic potential. At sufficiently small $\chi$ the quadratic mass term started to dominate and $\chi$ moved from the quartic valley to the quadratic one gaining nonzero and typically large angular momentum. This process occurred if the quartic and quadratic flat directions are different. This surely happens if $\alpha \neq 0$ but may also happen even with $\alpha=0$ if initially $\chi$ was in a different quartic valley from that of the quadratic $U_{m}(\chi)(2)$.
If the CP-odd phase $\alpha$ is small but non-vanishing, both baryonic and antibaryonic regions are possible with dominance of one of them. Matter and antimatter domain may exist but globally $B \neq 0$.

In ref. [1] a general renormalizable coupling of $\chi$ to the inflaton field $\Phi$ was introduced:

$$
\delta U=g|\chi|^{2}\left(\Phi-\Phi_{1}\right)^{2},
$$

where $g$ is a dimensionless coupling constant and $\Phi_{1}$ is a value of the inflaton which it passed during inflation. This potential looks as a very special one, but it is not so. It contains three renormalizable contributions: quartic, cubic, and quadratic terms. The only mild tuning of this potential is the value of $\Phi_{1}$.

With the new term and with an account of the so called Coleman-Weinberg contribution [9], arising from one-loop radiation corrections, the total potential governing the evolution of $\chi$ takes the form:

$$
\begin{aligned}
U & =g|\chi|^{2}\left(\Phi-\Phi_{1}\right)^{2}+\lambda|\chi|^{4} \ln \left(\frac{|\chi|^{2}}{\sigma^{2}}\right) \\
& +\lambda_{1}\left(\chi^{4}+\text { h.c. }\right)+\left(m^{2} \chi^{2}+\text { h.c. }\right) .
\end{aligned}
$$

The shape of this potential as a function of $|\chi|$ for different values of $\Phi$ is presented in Fig. 2.

When $\Phi$ is close to $\Phi_{1}$, the window to the flat directions is open but only for a relatively short period. At this stage cosmologically small but possibly astronomically large bubbles with high values of $\chi$ could be created. Later these high $\chi$ regions would create bubbles with very high baryonic number density occupying a small fraction of the universe volume, while the rest of the universe would have the normal baryon asymmetry $\beta \approx 6 \cdot 10^{-10}$, created in the dominant part of the universe with small $\chi$. The process when $\chi$ reaches large value but with low probability can be called phase transition of $3 / 2$ order, since in a sense such a behavior is between first and second order phase transitions.

The density contrast between the bubbles with high baryonic density (high B bubbles or HBB) and the low B average cosmological background is initially small, since before the QCD phase transition quarks populating the cosmological plasma are essentially massless, so the initial density perturbations are predominantly isocurvature. However, after the phase transition to the quark confinement phase quarks turned into heavy nucleons and the density contrast between $\mathrm{HBB}$ and the rest of the universe became large. Note that the contribution of HBBs into the total cosmological energy/mass density can be higher than the contribution of the normal free baryons.

The high density contrast, which appeared after the QCD phase transition, could lead to an early formation of compact stellar-type objects and possibly to a comparable amount of anti-objects, such that the bulk of baryons and (equal) antibaryons are in the form of compact stellarlike objects or $\mathrm{PBH}$, plus the sub-dominant observed homogeneous baryonic background. The amount of antimatter may be comparable or even larger than of the known baryons, but such "compact" (anti)baryonic objects do not contradict any existing observations $[5,7]$. 
The distributions of high baryon density bubbles over length and mass have log-normal form [1]:

$$
\frac{d N}{d M}=C_{M} \exp \left[-\gamma \ln ^{2}\left(M / M_{0}\right)\right]
$$

where $C_{M}, \gamma$, and $M_{0}$ are constant parameters. The spectrum is practically model independent, it is basically determined by inflation.

Adjusting parameters of the mass spectrum (7) using available astronomical data and constraints, we can predict the density of PBHs produced by the considered here mechanism. The prediction as a function of the black hole mass together with the existing observational bounds is presented in Fig. 3.

\section{Problems in contemporary and near contemporary universe.}

\subsection{Dense population of $z=5-10$ universe}

Astronomical data accumulated during the last few years have revealed that the early, $z \sim 10$, universe is unexpectedly dense, populated by the evolved objects which demand much more time for their creation than was available at that high redshifts. Among them there are bright but too young galaxies, QSO/supermassive $\mathrm{BHs}$, and gammabursters (supernovae). Moreover, the early universe contains much more dust than can be reasonably expected. A more detailed review and the list of literature is presented in ref. [4]. Here we discuss only the most striking creatures.

About 40 quasars with $z>6$ are already known, each quasar containing BH with $M \sim 10^{9} M_{\odot}$. Such black holes, when the Universe was less than one billion years old, present substantial challenges to theories of the formation and growth of black holes and the coevolution of black holes and galaxies. Even the origin of supermassive black holes in contemporary universe, which had 14 Gyr for their creation, is difficult to explain.

Very recently a new monster was discovered "An ultraluminous quasar with a twelve billion solar mass black hole at redshift 6.30" [10]. There is already a serious problem with formation of ten times lighter and less luminous quasars, which is multifold deepened with this new "creature". A formation of the new one with $M \approx 10^{10} M_{\odot}$ is absolutely impossible in the standard approach.

The premature appearance of supermassive black holes at $z \sim 6$ emerged as a great surprise. It is very difficult to understand how $10^{9} M_{\odot}$ black holes appeared so quickly after the big bang without invoking non-standard accretion physics and the formation of massive seeds, both of which are not observed in the local Universe [11]. On the other hand, such massive seeds are exactly the objects which are supplied by the HBBs discussed in the previous section.

Several galaxies have been observed at high redshifts, with natural gravitational lens "telescopes. Their creation was also unexpectedly early. E.g. a galaxy at $z \approx 9.6$ was discovered [12], which was created when the universe was about 0.5 Gyr old. Moreover a galaxy at $z \approx 11$ has been observed [13], which was formed earlier than the universe age was $0.41 \mathrm{Gyr}$ (or even shorter with larger $\mathrm{H}$ advocated by the recent traditional astronomical measurements).

An observation of not so young but extremely luminous galaxy is reported [14]. Its luminosity is thousand times higher than that of the Milky way: $L=3 \cdot 10^{14} L_{\odot}$, while the age is only $\sim 1.3$ Gyr. The galactic seeds, or embryonic black holes, might be bigger than thought possible. According to one of the authors of the discovery P. Eisenhardt: "How do you get an elephant? One way is start with a baby elephant." However, the origin of the baby elephant" is mysterious. The seed black hole should be already billions of solar masses, when our universe was only a tenth of its present age of 13.8 billion years. There is no way to create such a seed with standard mechanisms.

The medium around the observed early quasars contains considerable amount of "metals" (elements heavier than $\mathrm{He}$ ). According to the standard picture, only elements up to ${ }^{4} \mathrm{He}$ and traces of $\mathrm{Li}, \mathrm{Be}, \mathrm{B}$ were formed by the big bang nucleosynthesis, while heavier elements were created much later by stellar nucleosynthesis and dispersed in the interstellar space by supernova explosions. It already demands non-negligible time.

Much later molecular dust could form. Nevertheless it was discovered recently that the young universe at $z>6$ is quite dusty [15]. Dusty galaxies show up at redshifts corresponding to a Universe which is only about 500 Myr old. The highest redshift such object, HFLS3, lies at $\mathrm{z}=6.34$ and numerous other sources have been found [16].

Hence, prior to or simultaneously with the QSO formation a rapid star formation should take place. These stars should evolve to a large number of supernovae enriching interstellar space by metals through their explosions which later make molecules and dust. (We all are dust from SN explosions, but probably at much later time.)

Another possibility is a non-standard BBN due to very high baryonic density, which allows for formation of heavy elements beyond lithium.

Observations of high redshift gamma ray bursters (GBR) also indicate a high abundance of supernova at large redshifts. The highest redshift of the observed GBR is 9.4 and there are a few more GBRs with smaller but still high redshifts. The necessary star formation rate for explanation of these early GBRs is at odds with the canonical star formation theory.

All such early supernovae can be HBBs which were not massive enough to make PBHs but some compact stellar like objects which is also possible according to the discussed in sec. 3 scenario.

\subsection{Back to the future.}

Similar and possibly related mysteries exist in contemporary and near-contemporary universe.

An accumulation of quasars is discovered in a narrow spot in the sky [17]. at redshift $z \approx 2$. According to the authors of the paper: We discovered a physical association of four quasars embedded in a giant nebula. Quasars are rare 


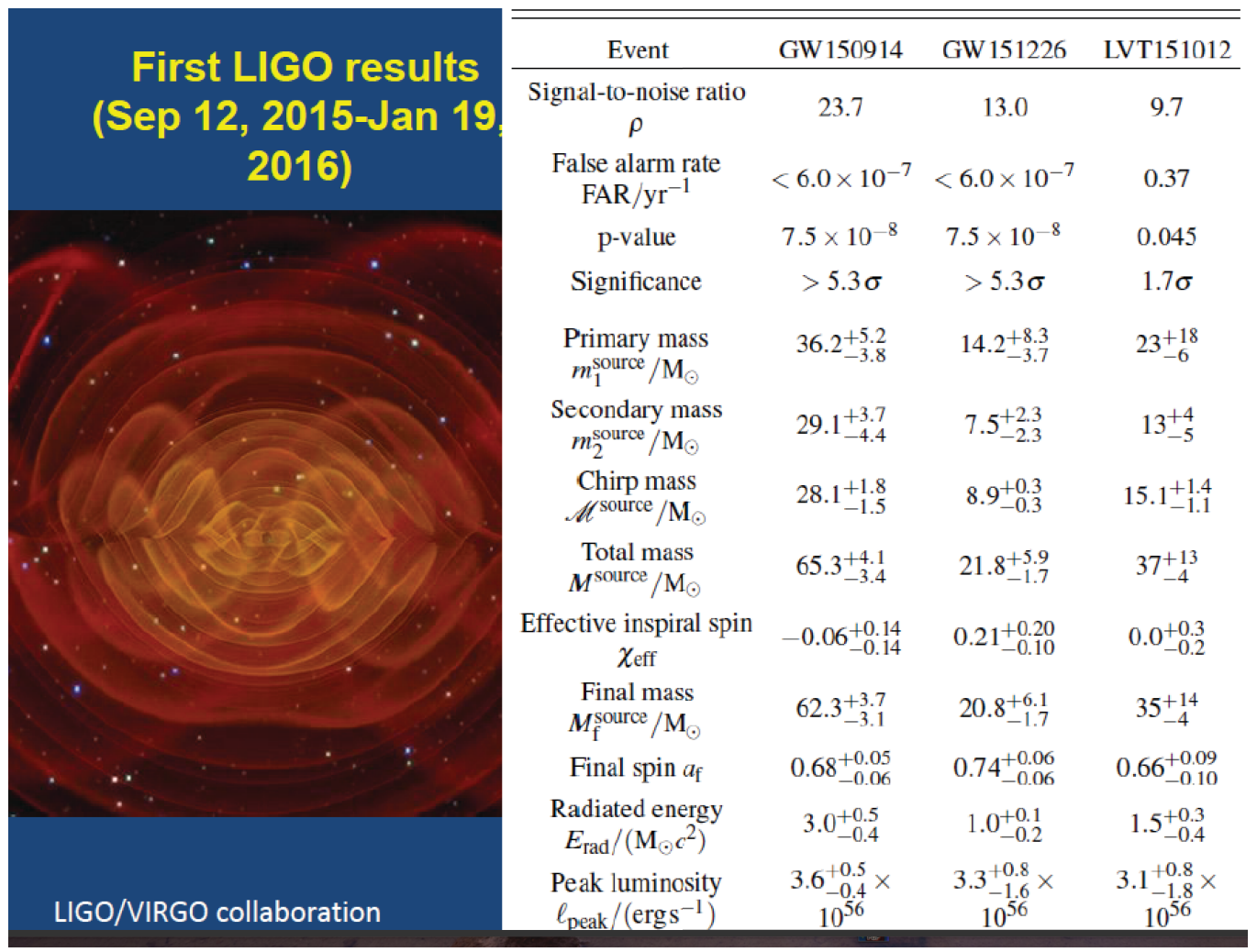

Figure 1. Three registered gravitational wave events.

objects separated by cosmological distances, so the chance of finding a quadruple quasar is $\sim 10^{-7}$. It implies that the most massive structures in the distant universe have a tremendous supply $\left(\sim 10^{11} M_{\odot}\right)$ of cool dense $\left(n \approx 1 / \mathrm{cm}^{3}\right)$ gas, in conflict with current cosmological simulations."

Every large galaxy and some smaller ones contain central supermassive BHs whose masses are larger than $10^{9} M_{\odot}$ in giant elliptical and compact lenticular galaxies and $\sim 10^{6} M_{\odot}$ in spiral galaxies like Milky Way. The origin of these superheavy BHs is not understood. Moreover, SHBs are observed in several small galaxies, where is no material to make a supermassive $\mathrm{BH}$.

It is intriguing if the type of the galaxy is determined by the mass of the original black hole seed.
The mass of $\mathrm{BH}$ is typically $0.1 \%$ of the mass of the stellar bulge of galaxy but some galaxies may have huge BH: e.g. NGC 1277 has the central BH of $1.7 \times 10^{10} M_{\odot}$, or $60 \%$ of its bulge mass [18]. This fact creates serious problems for the standard scenario of formation of central supermassive BHs by accretion of matter in the central part of a galaxy.

Report of the similar observations can be found in ref. [19]. The authors conclude that although supermassive black holes correlate well with their host galaxies, there is an emerging view that outliers exist. Henize 2-10, NGC 4889, and NGC1277 are examples of supermassive BHs at least an order of magnitude more massive than their host 


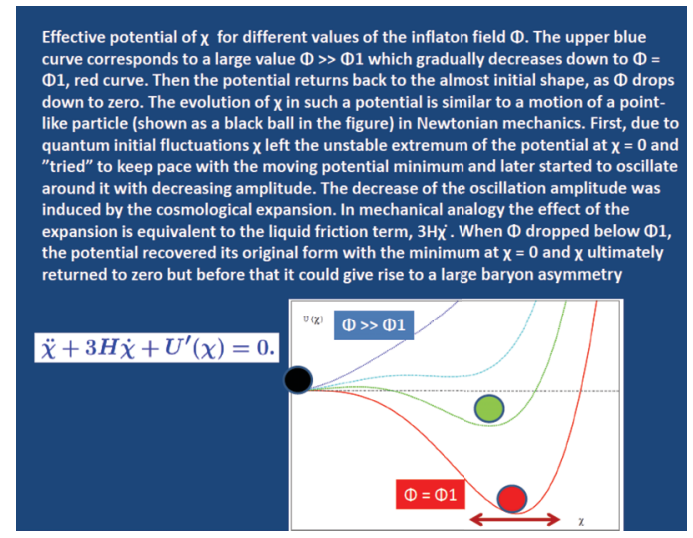

Figure 2. Evolution of the potential (6) as a function of $\chi$ for different values of $\Phi$.

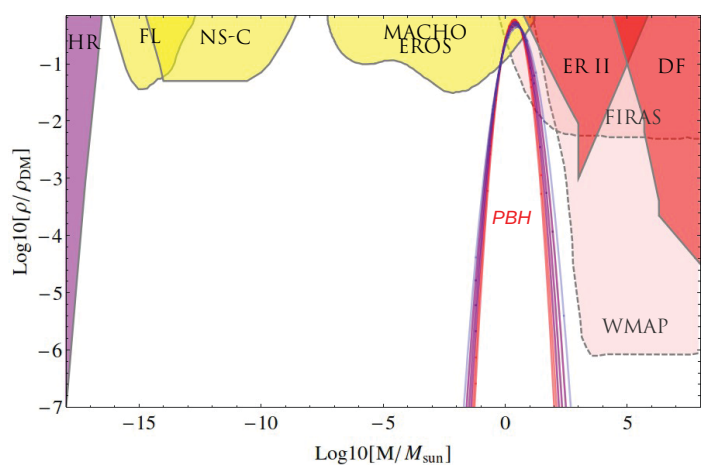

Figure 3. Constraints on $\mathrm{PBH}$ fraction in $\mathrm{DM}, f=\rho_{\mathrm{PBH}} / \rho_{\mathrm{DM}}$, where the PBH mass distribution is taken as $\rho_{\mathrm{PBH}}(M)=$ $M^{2} d N / d M$. The existing constraints (extragalactic $\gamma$-rays from evaporation (HR), femtolensing of $\gamma$-ray bursts (F), neutron-star capture constraints (NS-C), MACHO, EROS, OGLE microlensing (MACHO, EROS) survival of star cluster in Eridanus II (E), dynamical friction on halo objects (DF), and accretion effects (WMAP, FIRAS)). The PBH distribution is shown for ADBD parameters $\mu=10^{-43} \mathrm{Mpc}^{-1}, M_{0}=\gamma+0.1 \times \gamma^{2}-0.2 \times \gamma^{3}$ with $\gamma=0.75-1.1$ (red solid lines), and $\gamma=0.6-0.9$ (blue solid lines).

galaxy suggests. The dynamical effects of such ultramassive central black holes is unclear.

Several more observations of too heavy black holes in poor galaxies are presented in ref. [4] but the most striking one is given in the recent publication [20], where a supermassive $\mathrm{BH}$ was observed in practically empty space

Thus the inverted picture is more plausible: first a supermassive black hole was formed and attracted matter serving as a seed for subsequent galaxy formation $[1,21]$.

\subsection{Old stars, black holes, and MACHOS in the Milky Way}

Some more, at first sight unrelated, but probably the same kind problems are demonstrated by the contemporary universe. There are stars in the Milky Way, older than the Galaxy and even older than the universe (more than two sigma) and even one very old rocky planet. The mass distribution of black holes in the Galaxy and abundant MACHOs are also at odds with the standard astrophysics.

The new recently developed precise measurements allowed to determine age of several stars in the Milky Way with unprecedented accuracy. The results showed that quite a few stars are consuderably older than it was earlier expected.

Employing thorium and uranium abundances in comparison with each other and with several stable elements the age of metal-poor, halo star $\mathrm{BD}+17^{\circ} 3248$ was estimated as $13.8 \pm 4 \mathrm{Gyr}[22]$. For comparison the age of inner halo of the Galaxy $11.4 \pm 0.7$ Gyr [23]

The age of a star in the galactic halo, HE 1523-0901, was estimated to be about 13.2 Gyr [24]. First time many different chronometers, such as the U/Th, U/Ir, Th/Eu, and $\mathrm{Th} / \mathrm{Os}$ ratios to measure the star age have been employed.

Even more striking, the metal deficient high velocity subgiant in the solar neighborhood HD 140283 has the age $14.46 \pm 0.31$ Gyr [25]. The central value exceeds the universe age by two standard deviations, if $H=67.3$ $\mathrm{km} / \mathrm{sec} / \mathrm{Mpc}$ and $t_{U}=13.8 \mathrm{Gyr}$. The excess is even bigger for $H=74 \mathrm{~km} / \mathrm{sec} / \mathrm{Mpc}$, when $t_{U}=12.5 \mathrm{Gyr}$.

A discovery of a surprisingly old planet was recently announced [26] Its age is estimated as $10.6_{-1.3}^{+1.5}$ Gyr. (The age of the Earth: 4.54 Gyr.) A supenova explosion must precede formation of this planet.

The considered in this report scenario of HBB formation may explain these striking discoveries, because if the initial chemical content of a star is different from the canonical one, it may look older than it is in reality.

Some more mysteries are revealed by the black holes observed in the Galaxy. It was found that the $\mathrm{BH}$ masses are concentrated in the narrow range $(7.8 \pm 1.2) M_{\odot}[27]$.

This result agrees with another paper where a peak around $8 M_{\odot}$, a paucity of sources with masses below $5 M_{\odot}$, and a sharp drop-off above $10 M_{\odot}$ are observed [28].

These features are not expected in the standard model, but may fit our model with the log-normal mass spectrum of PBHs.

HBB can be also found by the effect of gravitational microlensing which may be caused by both visible and invisible stars. These objects are called Machos for Massive Astrophysical Compact Halo Objects. The observational situation with them is reviewed in ref. [5], where it is concluded that this population of the old invisible stars that evaded detection can well be HBBs discussed here. Those stars should be older than any kind of the oldest standard stellar populations. They will meet both criteria: they should be very weak and their cloud should have such a high velocity dispersion as needed. There is an intriguing possibility that some of those HBBs can be antistars in the Galaxy and in the galactic halo. 


\section{Conclusion}

1. Supersymmetric baryogenesis could lead to abundant formation of PBHs and compact stellar-like objects in the early universe after the QCD phase transition at $z \sim 10^{12}$ or $t \gtrsim 10^{-5} \mathrm{sec}$.

2. These objects have log-normal mass spectrum.

3. Adjusting the spectrum parameters is possible to explain the peculiar features of the sources of the gravitational waves observed by LIGO.

4. The considered mechanism solves the numerous mysteries of $z \sim 10$ universe: abundant population of supermassive black holes, early created gamma-bursters and supernovae, early bright galaxies, and evolved chemistry including dust.

5. There is persuasive data in favor of the inverted picture of galaxy formation, when first a supermassive $\mathrm{BH}$ seeds are formed and later they accrete surrounding matter forming galaxies.

6. An existence of supermassive black holes observed in all large and some small galaxies and even in almost empty environment is naturally explained.

7. "Older than the universe" stars may exist.

8. Existence and high density of invisible "stars" (machos) can be understood.

9. Some noticeable fraction of dark matter or even all of it can be made of PBHs.

10. Large amount of astronomical data the data strongly demand abundant cosmological population of $\mathrm{PBH}$ with wide mass spectrum.

Testable predictions:

A. Rate and masses of the BH sources of the coming GW events.

B. Possible existence of antimatter in our neighborhood, even in the Galaxy.

\section{Acknowledgement}

This work was supported by the Grant of President of Russian Federation for the leading scientific schools of the Russian Federation, NSh-9022.2016.2.

\section{References}

[1] A. Dolgov and J. Silk, Phys.Rev. D47, 4244 (1993); A.D. Dolgov, M. Kawasaki, and N. Kevlishvili, Nucl.
Phys. B807, 229 (2009).

[2] B. P. Abbott et al, Phys. Rev. Lett. 116, 061102 (2016).

[3] S. Blinnikov, A. Dolgov, N.K. Porajko, and K. Postnov, JCAP 1611, 036 (2016).

[4] A.D. Dolgov, Int. J. Mod. Phys. A31, 1645029 (2016); A.D. Dolgov, arXiv:1605.06749, Conference: C16-0216.1.

[5] S.I. Blinnikov, A.D. Dolgov, and K.A. Postnov, Phys. Rev. D92, 023516 (2015).

[6] A.D. Dolgov and S.I. Biinnikov, Phys. Rev. D89 2, 021301 (2014).

[7] C. Bambi and A. D. Dolgov, Nuclear Physics B 784, 132 (2007).

[8] I. Affleck and M. Dine, Nucl. Phys. B 249, 361 (1985).

[9] S.R. Coleman and E. Weinberg, Phys. Rev. D7, 1888 (1973).

[10] Xue-Bing Wu et al, Nature 518, 512 (2015).

[11] F. Melia, Astron. J. 147, 120 (2014).

[12] W. Zheng, et al, Nature 489, 406 (2012).

[13] D. Coe et al Astrophys. J. 762, 32 (2013).

[14] Chao-Wei Tsai, P.R.M. Eisenhardt et al, arXiv:1410.1751, Ap.J. in press.

[15] M. Mancini et al 1505.01841.

[16] L. Mattsson, arXiv:1505.04758v1.

[17] J.F. Hennawi et al, Science, 348, 779 (2015).

[18] R. C. E., Gebhardt et al, Nature, 491, 729 (2012).

[19] F. Khan, K. Holley-Bockelmann, P. Berczik, arXiv:1405.6425v1.

[20] J. J. Condon, J. Darling, Y. Y. Kovalev, L. Petrov, arXiv:1606.04067.

[21] R. C. E. van den Bosch et al, Nature, 491, 729 (2012)

[22] J.J. Cowan et al, Ap.J. 572, 861 (2002).

[23] J. Kalirai, Nature 486, 90 (2012).

[24] A. Frebe, N. Christlieb, J.E. Norris, C. Thom Astrophys. J. 660, L117 (2007).

[25] H. E. Bond et l Astrophys. J. Lett. 765, L12 (2013)/

[26] X. Dumusque, et al Ap. J., 789, 154 (2014).

[27] F. Ozel et al Astrophys. J. 725, 1918 (2010).

[28] L. Kreidberg et al Astrophys. J. 757, 36 (2012). 